\title{
Study of atomic transition self-broadening and shift with the help of a nano-cell
} \author{
and G Pichler ${ }^{2}$ \\ ${ }^{1}$ Institute for Physical Research, NAS of Armenia, Ashtarak-2, Armenia \\ ${ }^{2}$ Institute of Physics, Bijenička 46, POB 304, HR-10001 Zagreb, Croatia
}

T Varzhapetyan ${ }^{1}$, A Nersisyan ${ }^{1}$, V Babushkin ${ }^{1}$, D Sarkisyan ${ }^{1}, \mathbf{S}$ Vdović $^{2}$

Received 27 September 2007, in final form 22 July 2008

Published 3 September 2008

Online at stacks.iop.org/JPhysB/41/185004

\begin{abstract}
We demonstrate experimentally that it is possible to detect and measure the self-broadening rate and frequency shift of the rubidium second principal series atomic transitions at very high atomic vapour densities up to $\sim 10^{17} \mathrm{~cm}^{-3}$. The experimental procedure includes the usual absorption measurement technique and a sealed-off nano-cell containing $\mathrm{Rb}$ vapour operating at high temperatures with a thickness of $L=\lambda / 2$, where $\lambda$ is the resonant wavelength

$(420 \mathrm{~nm})$.
\end{abstract}

\section{Introduction}

Laser spectroscopy in a thin film of alkali atomic vapour with thickness in the range of $10-1000 \mu \mathrm{m}$ has provided a novel method for sub-Doppler spectroscopy [1]. Recently developed nano-cells (or so-called extremely thin cells, ETC) of submicron thickness $L$ of atomic vapour [2] allows one to study the peculiarities of resonant absorption and fluorescence for the case when $L$ is of the order of a laser resonant wavelength $\lambda$. It has been shown that the coherent response of atomic dipoles to light results in appreciable narrowing of the hyperfine transition profile (which is several times narrower than the Doppler profile of the atomic transition) when the cell thickness is $L=\lambda / 2$ [3]. It has been revealed that for a low laser intensity $\left(<1 \mathrm{~mW} \mathrm{~cm} \mathrm{~cm}^{-2}\right)$, the linewidth of the absorption line at the hyperfine transition exhibits oscillatory behaviour and has a minimum value when $L=(2 n+1) \lambda / 2$ and a maximum value when $L=n \lambda$ ( $n$ being an integer). This is the manifestation of the so-called collapse and revival of the Dicke-type coherent narrowing effect [3,4]. Also, some other interesting peculiarities have been observed with the use of nano-cells; particularly, the transmission and the fluorescence do not have 'reciprocal' behaviour.

It is interesting to note that in the case of the cell thickness $L=\lambda / 2$, the atomic vapour density can be increased up to $10^{16}-10^{17} \mathrm{~cm}^{-3}(0.58-5.8$ Torr) while still having an absorption below $100 \%$ (this depends on the atomic transition probability). This is simply because of the product $N \cdot L$, which stays below the 'complete absorption' value for really high vapour densities due to the small value of $L$. Thus, with the help of the nano-cell, simply by detecting transmission spectra, it might be possible to study a shift and broadening of atomic transitions at very high atomic densities.

Here we report on the experimental results of the selfbroadening and frequency shift studies of the Rb $420 \mathrm{~nm}$ second principal series atomic line. The experiment was carried out using a nano-cell with a thickness of $L=\lambda / 2=$ $210 \mathrm{~nm}$. In the case of the first principal series resonance lines, the resonance interaction $\left( \pm C_{3} / R^{3}\right)$ leads to self-broadening only, without the frequency shift. However, at the second principal series lines, the resonance interaction is very weak due to much smaller oscillator strength, and the van der Waals (vdW) interaction $\left(-C_{6} / R^{6}\right)$ becomes stronger giving rise to both broadening and shift.

Note that the frequency shift and broadening of atomic transitions caused by the buffer gas can be measured using heat-pipe ovens (or sealed-off cells) of usual length $L>$ $1 \mathrm{~cm}$ as described in [5]. Self-broadening and shift become pronounced at atomic vapour densities $N>10^{14} \mathrm{~cm}^{-3}$ (0.0058 Torr) when on-resonance absorption (on D1 and D2 first resonance lines) in atomic vapours confined in ordinary $\mathrm{cm}$-sized cells is about $100 \%$. One of the widely used methods employed for broadening and shift measurements at high atomic densities is selective reflection (SR) spectroscopy [6]. But for correct measurement of broadening and shift rates in the case of the SR technique the spectra must undergo further non-trivial processing.

By using the nano-cell, it is now possible to directly measure the broadening and shift of the second resonance 


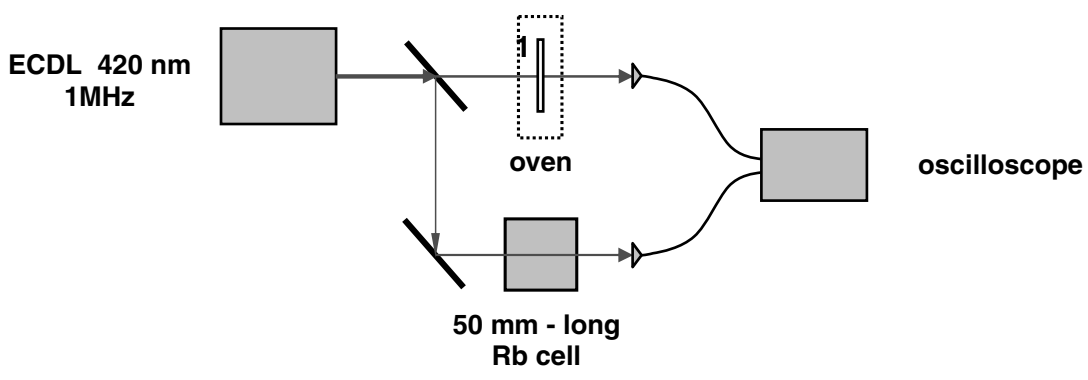

Figure 1. Experimental setup: (1) the nano-cell inside the oven.

$\mathrm{Rb}$ atomic line at atomic densities unattainable in normal cells or heat-pipe oven cells.

\section{Experiment}

The construction of the nano-cell filled with $\mathrm{Rb}$ is similar to that described in [2]. In our case, garnet plates of $\sim 2 \mathrm{~mm}$ thickness were used as windows of the cell. The diameter of the cell windows was $30 \mathrm{~mm}$. In order to provide high number density of $\mathrm{Rb}$ atomic vapour, the cell was placed in a specially designed oven where temperatures of the body and the side arm of the cell could be increased up to $450{ }^{\circ} \mathrm{C}$ and controlled independently. The thickness of the nano-cell was measured by employing an interferometric method also described in [3] using the resonant laser radiation and found to be in the range 50-900 nm (in the whole aperture of the cell windows).

The experimental arrangement is shown in figure 1. An external cavity diode laser system (ECDL) Toptica DL100 equipped with the NLHV3000E laser diode (TOPTICA, NICHIA) with radiation wavelength at $420 \mathrm{~nm}$ was used in our measurements. The linewidth of the laser radiation was $\sim 1 \mathrm{MHz}$. The laser beam diameter was $\sim 1 \mathrm{~mm}$ corresponding to the area of the cell window in which $L=\lambda / 2$ is valid. The output power of the laser was reduced using a neutral density filter down to $15 \mathrm{~mW} \mathrm{~cm} \mathrm{~cm}^{-2}$ (measured before entering the sample cell) to avoid saturation effects. The laser radiation frequency was scanned in the vicinity of the $5^{2} \mathrm{~S}_{1 / 2}-6^{2} \mathrm{P}_{3 / 2}$ violet transitions $(420.2 \mathrm{~nm})$ of the ${ }^{85} \mathrm{Rb}$ and ${ }^{87} \mathrm{Rb}$ isotopes (in natural mixture) with the $0.7 \mathrm{GHz} \mathrm{ms}{ }^{-1}$ scanning rate. Signals from the two photodiodes were recorded with a two-channel analogue-digital oscilloscope connected to the laboratory computer. Due to the original construction, the density (as well as the pressure) of $\mathrm{Rb}$ atomic vapour is determined by the temperature of the boundary of the $\mathrm{Rb}$ metal in the side arm which is determined via one of the Chromel-Alumel thermocouples (CAT). This thermocouple is tied close to the boundary of the $\mathrm{Rb}$ metal column in the side arm in order to realize a mechanical contact with the sapphire tube of the side arm. In this way, we ensured accurate temperature readings essential for the correct density and pressure determination which is the major cause of uncertainties in the analysis.

\section{Results}

The transmission spectra of the $\mathrm{Rb}$ atomic vapour column at the thickness $L=\lambda / 2(210 \mathrm{~nm})$ were recorded for temperatures from $257{ }^{\circ} \mathrm{C}$ up to $383{ }^{\circ} \mathrm{C}$. Simultaneously, the transmission spectra of a $50 \mathrm{~mm}$ glass cell at $\sim 30{ }^{\circ} \mathrm{C}$ filled with $\mathrm{Rb}$ was recorded which served as reference spectra (figure 7). All reported density values are derived from the Nesmeyanov vapour pressure curves [7] using the temperature of the side arm of the cell (cold finger). The densities were verified by comparing low intensity peak absorption values in the nano-cell and in a room temperature $50 \mathrm{~mm}$ long cell. Unfortunately, the construction of our sapphire cell did not allow us to keep the rubidium vapour temperature constant, while simultaneously changing the cold finger temperature independently. Expressions for the impact theory line broadening when the broadening is due to the resonance interaction only indicate that the broadening rates are independent of the gas temperature $T$. However, if higher order non-resonant terms of the interatomic potential such as the $1 / R^{6} \mathrm{vdW}$ interaction are significant, then the collision-broadened profile, although still Lorentzian, will be shifted and will have a temperature-dependent broadening rate [8]. Impact theory gives a broadening rate that scales with temperature as $T^{0.3}$ [9] from which we estimate our error of obtained broadening rates connected with non-constant vapour temperature to be around $5 \%$. Here we note that earlier published measurements of self-broadening and shift of various alkali resonance lines using the selective reflection technique $[10,11]$ were performed under similar conditions in order to prevent atomic vapour condensation and determine correctly the vapour density. Moreover, we have already checked the transmission for the $780 \mathrm{~nm} \mathrm{Rb} \mathrm{D2}$ line at thickness $\lambda / 2$ by keeping the side arm temperature constant $\left(\sim 120^{\circ} \mathrm{C}\right)$ and varying the temperature of the cell body up to $250{ }^{\circ} \mathrm{C}$. We have detected a $10 \%$ broadening increase whereas the shift was absent.

Relevant atomic levels of $5^{2} \mathrm{~S}_{1 / 2}-6^{2} \mathrm{P}_{3 / 2}$ transitions for ${ }^{85} \mathrm{Rb}$ and ${ }^{87} \mathrm{Rb}$ are presented in figure 2 . As can be seen, the ground state hyperfine splitting is $3036 \mathrm{MHz}$ for ${ }^{85} \mathrm{Rb}$ and $6835 \mathrm{MHz}$ for ${ }^{87} \mathrm{Rb}$. The hyperfine splitting of the exited state $\left(6 \mathrm{P}_{3 / 2}\right)$ for both ${ }^{85} \mathrm{Rb}$ and ${ }^{87} \mathrm{Rb}$ due to the small values of the splitting is not resolved in an ordinary cell absorption spectrum. As was shown in [3, 4], the line shape of nano-cell on-resonance transmission spectra can be strongly modified by the Fabry-Perot nature of the cell. In our case all measurements were performed at the cell thickness $L=\lambda / 2$ at which the line shapes are not affected [3, 4]. Recorded transmission spectra at seven different temperatures of the cell body and side arm are presented in figure 3 . The absorption 

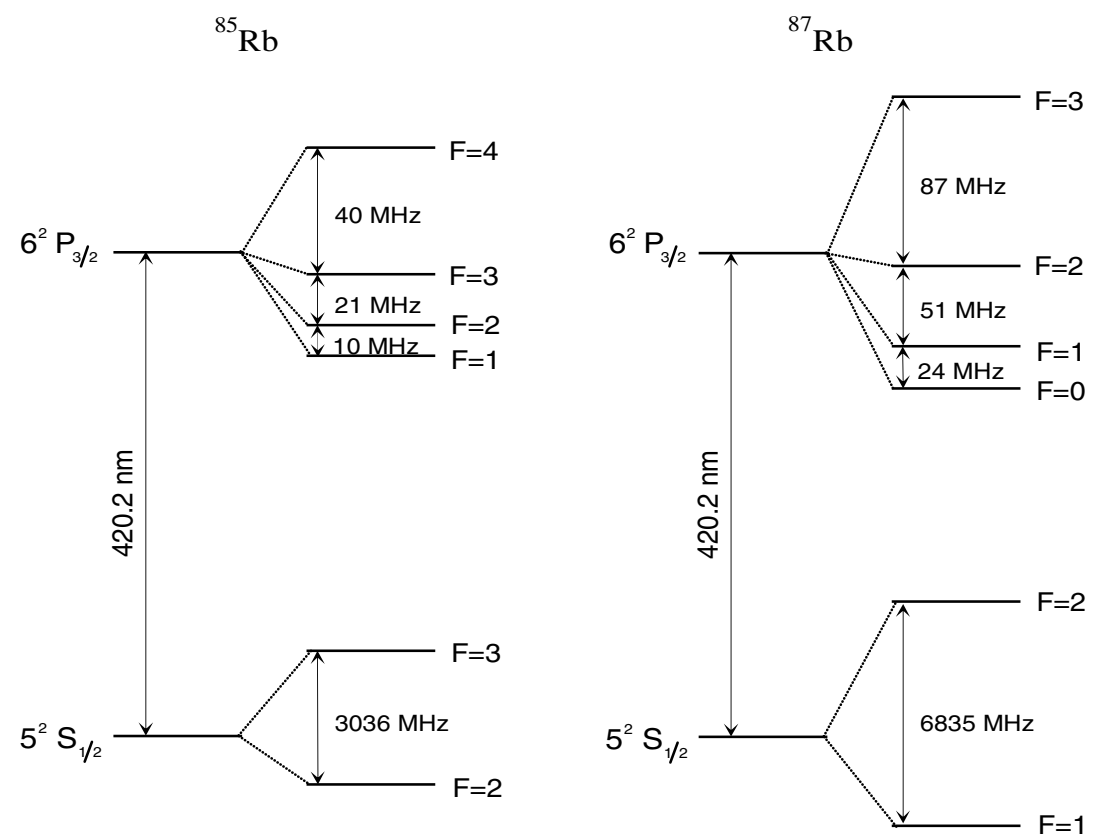

Figure 2. Energy level diagram of $5^{2} \mathrm{~S}_{1 / 2}-6^{2} \mathrm{P}_{3 / 2}$ transitions for ${ }^{85} \mathrm{Rb}$ and ${ }^{87} \mathrm{Rb}$.

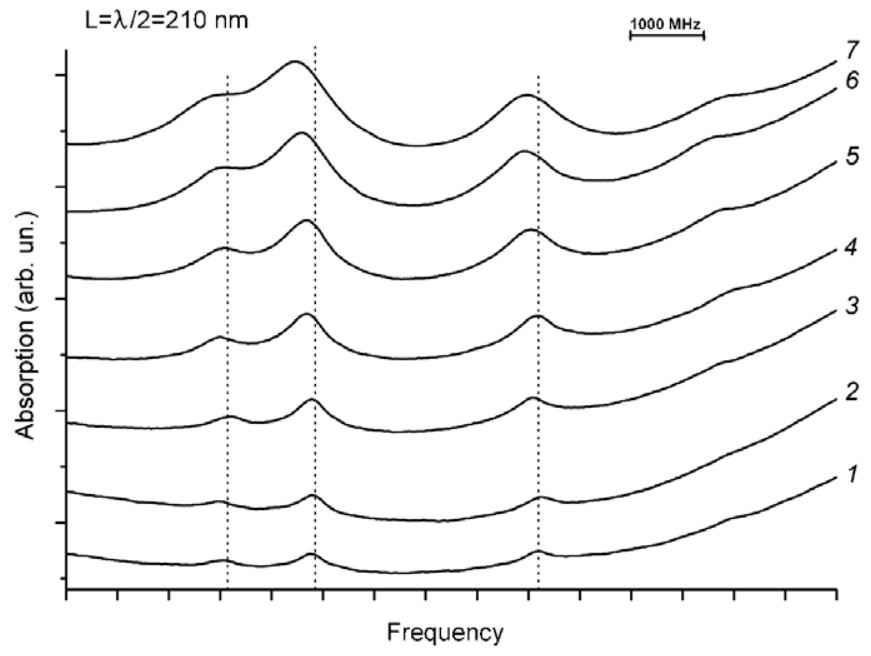

Figure 3. Absorption spectra of $420 \mathrm{~nm}$ wavelength radiation through ETC at the thickness $L=\lambda / 2=210 \mathrm{~nm}$ at different vapour densities (temperatures): (1) $257^{\circ} \mathrm{C}$ and $291^{\circ} \mathrm{C}$; (2) $279^{\circ} \mathrm{C}$ and $309^{\circ} \mathrm{C}$; (3) $305^{\circ} \mathrm{C}$ and $328^{\circ} \mathrm{C}$; (4) $322^{\circ} \mathrm{C}$ and $340{ }^{\circ} \mathrm{C}$; (5) $340{ }^{\circ} \mathrm{C}$ and $375{ }^{\circ} \mathrm{C}$; (6) $362{ }^{\circ} \mathrm{C}$ and $405{ }^{\circ} \mathrm{C}$; (7) $383{ }^{\circ} \mathrm{C}$ and $417^{\circ} \mathrm{C}$.

profiles consist of four lines- two (inner) resulting from ${ }^{85} \mathrm{Rb}$ and another two (outer) from ${ }^{87} \mathrm{Rb}$. Each line in these profiles corresponds to a set of transitions from one hyperfine level of the ground state $\left(5^{2} \mathrm{~S}_{1 / 2}\right)$ of one of the isotopes to the excited $6^{2} \mathrm{~S}_{1 / 2}$ hyperfine states.

In order to determine the self-broadening and shift parameters from the measured absorption spectra, the standard fitting procedure was employed. During the fitting (processing) procedure, the wavelength-dependent intensity was taken into account. We used Voigt profiles in the fitting procedure and these profiles were calculated for all 12 hyperfine transitions and added to give the total absorption spectrum (see figure 4 ). The ground and excited state hyperfine

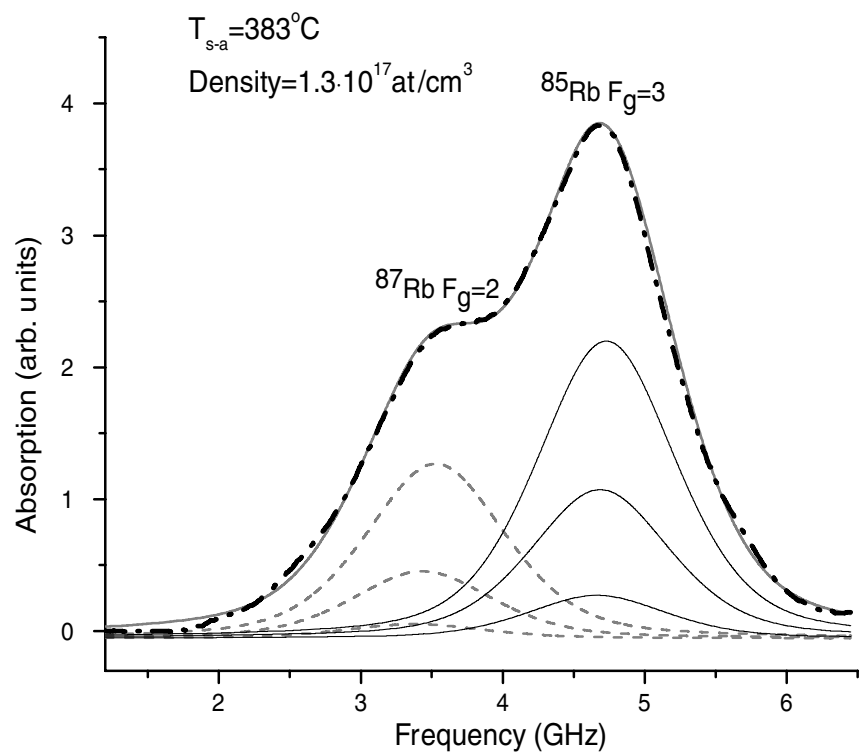

Figure 4. Example of the fits of the Voigt profiles to the experimental profile taking into account the excited levels' hyperfine structure.

splittings were taken as fixed parameters together with their transition probabilities (intensities). Free parameters include the spectral linewidth of transitions and their frequencies (shifts). This fitting procedure was performed for all spectra recorded at seven different temperatures (i.e. atomic vapour densities). Simple empiric formulae have been obtained for the data shown in figure 5:

$$
\begin{gathered}
{ }^{87} \mathrm{Rb} F_{\mathrm{g}}=1 \rightarrow F_{\mathrm{e}}=0,1,2 \\
\Delta v_{\mathrm{B}}=392[\mathrm{MHz}]+116[\mathrm{MHz} / \text { Torr }] \times p[\text { Torr }], \\
{ }^{85} \mathrm{Rb} F_{\mathrm{g}}=3 \rightarrow F_{\mathrm{e}}=2,3,4 \\
\Delta v_{\mathrm{B}}=411[\mathrm{MHz}]+88[\mathrm{MHz} / \text { Torr }] \times p[\text { Torr }],
\end{gathered}
$$




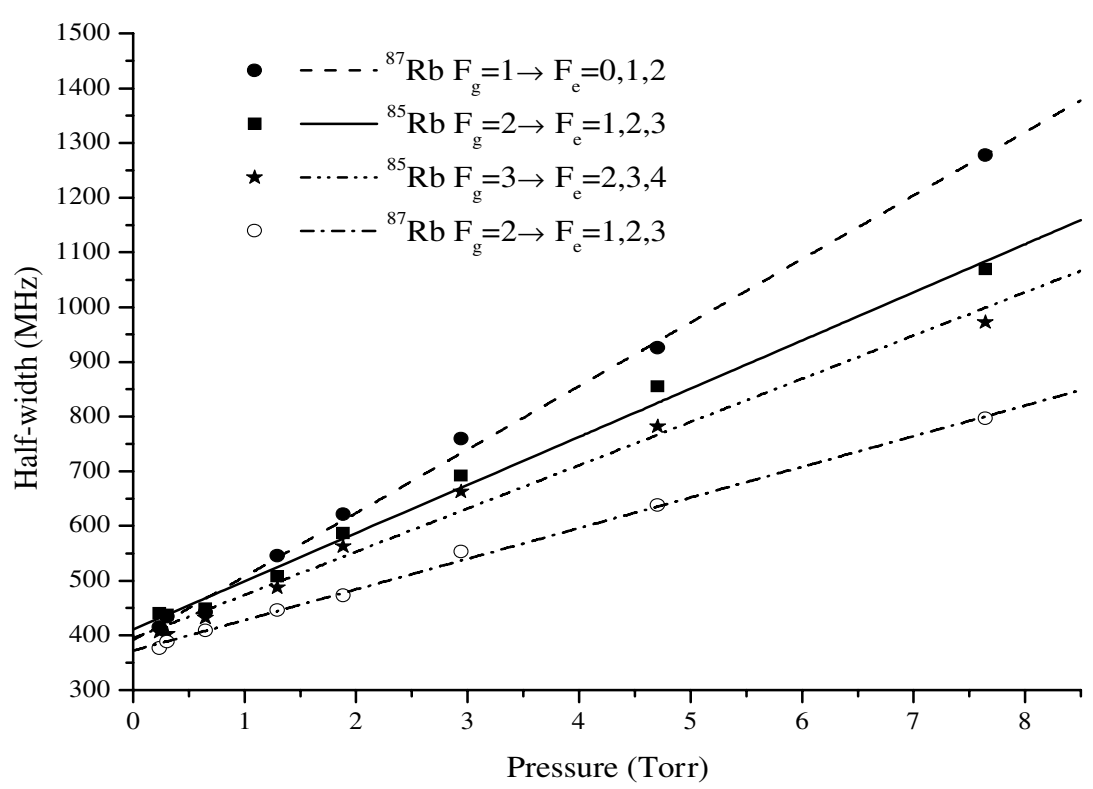

Figure 5. Dependences of $\mathrm{Rb}$ transitions $(420 \mathrm{~nm}$ ) spectral linewidths on the atomic vapour pressure.

$$
\begin{aligned}
& { }^{85} \mathrm{Rb} F_{\mathrm{g}}=2 \rightarrow F_{\mathrm{e}}=1,2,3 \\
& \Delta v_{\mathrm{B}}=395[\mathrm{MHz}]+79[\mathrm{MHz} / \text { Torr }] \times p[\text { Torr }], \\
& { }^{87} \mathrm{Rb} F_{\mathrm{g}}=2 \rightarrow F_{\mathrm{e}}=1,2,3 \\
& \quad \Delta v_{\mathrm{B}}=372[\mathrm{MHz}]+56[\mathrm{MHz} / \text { Torr }] \times p[\text { Torr }] .
\end{aligned}
$$

As was already mentioned, the absorption spectra of the $50 \mathrm{~mm}$ long $\mathrm{Rb}$ cell were recorded simultaneously as a reference spectrum. This spectrum was also fitted employing the same procedure with Voigt profiles and the obtained results were used to calibrate the frequency scale and calculate the frequency shifts.

Measured broadening rates are presented in figure 5. As can be seen from the figure, the most 'affected' transition is ${ }^{87} \mathrm{Rb} F_{\mathrm{g}}=1 \rightarrow F_{\mathrm{e}}=0,1,2$ with the broadening rate of $\sim 116 \mathrm{MHz}$ /Torr. The least affected is the ${ }^{87} \mathrm{Rb} F_{\mathrm{g}}=$ $2 \rightarrow F_{\mathrm{e}}=1,2,3$ transition with the broadening rate of $\sim 56 \mathrm{MHz} /$ Torr.

Frequency shift dependences on the atomic vapour density measured from the same spectra (figure 3) are shown in figure 6. Transitions from the $F_{\mathrm{g}}=2$ state of the ${ }^{85} \mathrm{Rb}$ isotope experience the largest frequency red shifts with increasing vapour pressure evaluated to $\sim 34.2 \mathrm{MHz}$ /Torr. Transitions from the $F_{\mathrm{g}}=3$ state of ${ }^{85} \mathrm{Rb}$ have $24.8 \mathrm{MHz} /$ Torr red shifts. Transitions from the $F_{\mathrm{g}}=2$ state of ${ }^{87} \mathrm{Rb}$ have $21.4 \mathrm{MHz} /$ Torr red shifts. All estimated errors of the calculated broadening and shift rates are presented in tables 1 and 2, respectively.

The absorption spectrum of the nano-cell with the thickness $L=\lambda / 2=210 \mathrm{~nm}$ (1) at the temperatures $T_{\mathrm{S}-\mathrm{A}}=$ $229{ }^{\circ} \mathrm{C}$ and $T_{\mathrm{W}}=276{ }^{\circ} \mathrm{C}$, and the absorption spectrum of the $50 \mathrm{~mm}$ glass cell at $\sim 30{ }^{\circ} \mathrm{C}$ (2) are presented in figure 7 . As can be seen, the linewidth of $\mathrm{Rb}$ atomic transitions at $420 \mathrm{~nm}$ obtained by using the nano-cell is much narrower than that obtained by an ordinary cell; thus, the transmission spectra of the nano-cell with $L=\lambda / 2$ can be used as a good frequency reference for this spectral region.

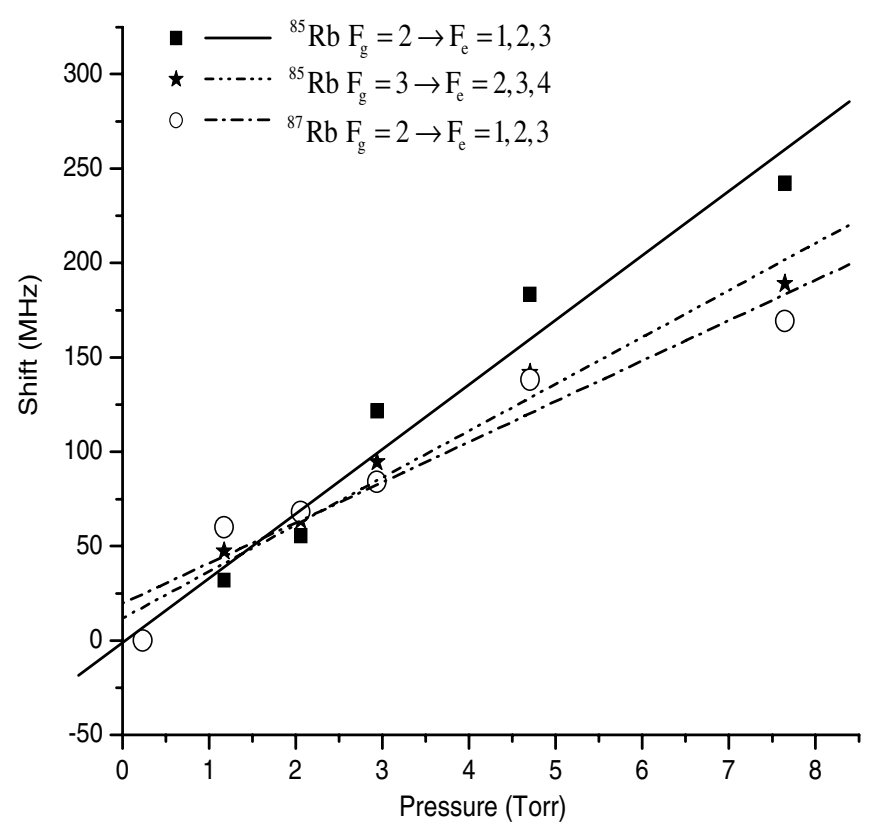

Figure 6. Transition frequency shift dependences on the atomic vapour pressure.

\section{Discussion}

It is important to note that another effect that may shift or broaden the observed violet lines, both in the transmission spectrum and in the fluorescence spectrum, is the long-range $\mathrm{vdW}$ attraction between the atom (in the ground or the excited states) and the dielectric surface (YAG windows in our case) [12]. It originates in the coupling between the quantum fluctuations of the atomic dipole and the instantaneously induced polarization of the surface, and scales like $1 / d^{3}$, with $d$ being the atom-surface distance. Indeed, a frequency shift and a lineshape distortion are currently observed in nano- 


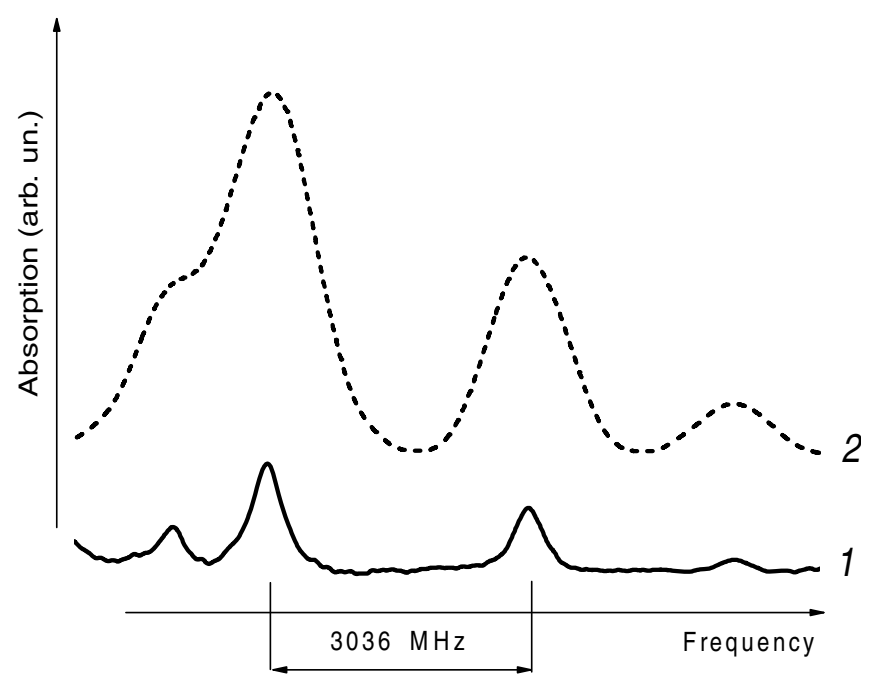

Figure 7. The comparison between absorption spectra of the nano-cell (1) (the thickness $L=\lambda / 2=210 \mathrm{~nm}, T_{\mathrm{S}-\mathrm{A}}=229^{\circ} \mathrm{C}$, $\left.T_{\mathrm{W}}=276{ }^{\circ} \mathrm{C}\right)$ and the $50 \mathrm{~mm}$ glass cell at $\sim 30^{\circ} \mathrm{C}(2)$.

Table 1. Calculated error of different hyperfine transition broadening rates.

\begin{tabular}{lllll}
\hline $\begin{array}{l}\text { Vapour } \\
\text { pressure } \\
\text { (Torr) }\end{array}$ & $\begin{array}{l}{ }^{87} \mathrm{Rb} ; F_{\mathrm{g}}=2 \\
(\mathrm{MHz})\end{array}$ & $\begin{array}{l}{ }^{85} \mathrm{Rb} ; F_{\mathrm{g}}=3 \\
(\mathrm{MHz})\end{array}$ & $\begin{array}{l}{ }^{85} \mathrm{Rb} ; F_{\mathrm{g}}=2 \\
(\mathrm{MHz})\end{array}$ & $\begin{array}{l}{ }^{87} \mathrm{Rb} ; F_{\mathrm{g}}=1 \\
(\mathrm{MHz})\end{array}$ \\
\hline 7.63 & 11 & 10.5 & 9 & 16 \\
4.71 & 10 & 10.5 & 9.5 & 14 \\
2.94 & 11 & 10 & 9 & 17 \\
1.88 & 10.5 & 11 & 10 & 16 \\
1.29 & 12 & 11 & 9.5 & 14 \\
0.65 & 12 & 10.5 & 9 & 16 \\
0.31 & 12 & 9.5 & 9.5 & 15 \\
\hline
\end{tabular}

Table 2. Calculated error of the different hyperfine transition shifts.

\begin{tabular}{lllll}
\hline \multirow{2}{*}{$\begin{array}{l}\text { Vapour } \\
\text { pressure } \\
\text { (Torr) }\end{array}$} & $\begin{array}{c}4 \\
{ }^{87} \mathrm{Rb} ; F_{\mathrm{g}}=2 \\
(\mathrm{MHz})\end{array}$ & $\begin{array}{l}{ }^{85} \mathrm{Rb} ; F_{\mathrm{g}}=3 \\
(\mathrm{MHz})\end{array}$ & $\begin{array}{l}{ }^{85} \mathrm{Rb} ; F_{\mathrm{g}}=2 \\
(\mathrm{MHz})\end{array}$ & $\begin{array}{l}{ }^{87} \mathrm{Rb} ; F_{\mathrm{g}}=1 \\
(\mathrm{MHz})\end{array}$ \\
\hline 7.63 & 12 & 10 & 9 & 12 \\
4.71 & 12.5 & 11 & 10 & 12 \\
2.94 & 11 & 9.5 & 11 & 14 \\
1.88 & 9 & 10 & 9.5 & 13 \\
1.29 & 10.5 & 9 & 9 & 15 \\
\hline
\end{tabular}

cells under various conditions with the help of transmission spectra. For the first $\mathrm{D}$ lines of $\mathrm{Rb}$ and $\mathrm{Cs}$ atoms, vdW attraction could be detected at the thickness below $100 \mathrm{~nm}$ by the red shift of resonant transmission spectra. For a higher atomic energy level, vdW attraction could be detected even at larger atom-wall distances. That is why the measured value of the frequency shift and broadening also contains some additional contribution from the influence of the atom-surface interaction. This interaction induces a red shift that is linear in atomic density [13].

Also, the calculated vapour pressures depend on the accuracy of the vapour pressure curves and the measured metal reservoir temperature. From [14] we deduced that a realistic

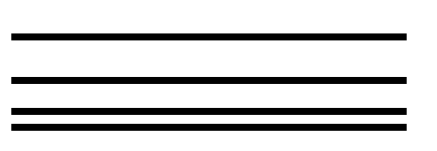

${ }^{87} \mathrm{Rb}(6 \mathrm{p}, \mathrm{F}=3)+{ }^{87} \mathrm{Rb}(5 \mathrm{~s}, \mathrm{~F}=2)$

${ }^{87} \mathrm{Rb}(6 \mathrm{p}, \mathrm{F}=2)+{ }^{87} \mathrm{Rb}(5 \mathrm{~s}, \mathrm{~F}=2)$

${ }^{87} \mathrm{Rb}(6 \mathrm{~s}, \mathrm{~F}=1)+{ }^{87} \mathrm{Rb}(5 \mathrm{~s}, \mathrm{~F}=2)$

${ }^{87} \mathrm{Rb}(6 \mathrm{~s}, \mathrm{~F}=0)+{ }^{87} \mathrm{Rb}(5 \mathrm{~s}, \mathrm{~F}=2)$

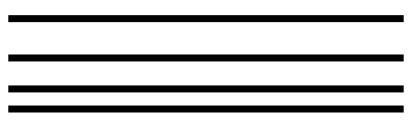

${ }^{87} \mathrm{Rb}(6 \mathrm{p}, \mathrm{F}=3)+{ }^{87} \mathrm{Rb}(5 \mathrm{~s}, \mathrm{~F}=1)$

${ }^{87} \mathrm{Rb}(6 \mathrm{p}, \mathrm{F}=2)+{ }^{87} \mathrm{Rb}(5 \mathrm{~s}, \mathrm{~F}=1)$

${ }^{87} \mathrm{Rb}(6 \mathrm{p}, \mathrm{F}=1)+{ }^{87} \mathrm{Rb}(5 \mathrm{~s}, \mathrm{~F}=1)$

${ }^{87} \mathrm{Rb}(6 \mathrm{p}, \mathrm{F}=0)+{ }^{87} \mathrm{Rb}(5 \mathrm{~s}, \mathrm{~F}=1)$

${ }^{87} \mathrm{Rb}(5 \mathrm{~s}, \mathrm{~F}=2)+{ }^{87} \mathrm{Rb}(5 \mathrm{~s}, \mathrm{~F}=2)$

${ }^{87} \mathrm{Rb}(5 \mathrm{~s}, \mathrm{~F}=2)+{ }^{87} \mathrm{Rb}(5 \mathrm{~s}, \mathrm{~F}=1)$

${ }^{87} \mathrm{Rb}(5 \mathrm{~s}, \mathrm{~F}=1)+{ }^{87} \mathrm{Rb}(5 \mathrm{~s}, \mathrm{~F}=2)$

\section{${ }^{87} \mathrm{Rb}(5 \mathrm{~s}, \mathrm{~F}=1)+{ }^{87} \mathrm{Rb}(5 \mathrm{~s}, \mathrm{~F}=1)$}

Figure 8. Partial energy diagram of asymptotic energies of the ${ }^{87} \mathrm{Rb}+{ }^{87} \mathrm{Rb}$ diatomic system relevant for the discussion of the broadening effects of the second resonance doublet.

uncertainty in the density would be $15 \%$. We note that, to the best of our knowledge, there are no available data on the self-broadening and shift of the rubidium second resonance doublet which could be used to be compared with the results presented in this work.

At higher densities the local field effect (Lorentz-Lorenz correction) is also present, shifting the resonance of the dielectric constant from that of the atomic polarizability by the amount $\Delta \omega_{L}$. At high vapour densities such that $N k^{-3} \geqslant 1$, the line shifts due to the Lorentz-Lorenz correction, the atomdielectric wall interaction and the non-exponential decay of the field in the vapour should be evaluated and the resonance shift value must be corrected for their contribution to the total shift [15].

It may be valuable to explain briefly the fact that we have different broadening rates of the hyperfine spectral components. It comes as a consequence of the complex structure of the interaction potential curves between the rubidium atoms as may be deduced from figures 6-8 in [16]. The authors discuss the case of the $R b(5 p)+R b(5 s)$ hyperfine interaction potential curves at asymptotic interatomic distances, just around atomic hyperfine levels. In [17], a similar complex structure of the hyperfine potential curves in the case of the $\mathrm{Na}(3 \mathrm{p})+\mathrm{Na}(3 \mathrm{~s})$ interaction is shown in figure 22. Unfortunately, there are no such theoretical calculations of the $\mathrm{Rb}(6 \mathrm{p})+\mathrm{Rb}(5 \mathrm{~s})$ hyperfine interaction potential curves. However, in figure 8 , we present a sketch of the asymptotic hyperfine energies pertinent to ${ }^{87} \mathrm{Rb}_{2}$ dimer. Out of each asymptote emerges a few or more of potential curves. The broadening rates could be in principle deduced 
from that complex structure of potential curves using impact broadening approximation. Since the potential curves are different at different hyperfine asymptotes, it is also expected that the broadening rates and shifts will be different for all hyperfine spectral lines studied in the present experiments. It is of considerable importance to mention that the ${ }^{87} \mathrm{Rb}(6 \mathrm{~s}, 6 \mathrm{p})+$ ${ }^{87} \mathrm{Rb}(5 \mathrm{~s})$ interaction is only one of the several important interactions in the thin cell. We have also the heteronuclear case ${ }^{87} \mathrm{Rb}(6 \mathrm{~s}, 6 \mathrm{p})+{ }^{85} \mathrm{Rb}(5 \mathrm{~s})$ and another homonuclear case ${ }^{85} \mathrm{Rb}(6 \mathrm{~s}, 6 \mathrm{p})+{ }^{85} \mathrm{Rb}(5 \mathrm{~s})$. In all those cases potential curves are different. Due to the recoupling of angular momenta, the hyperfine potential curves emerging from $6 p+5$ s asymptotes do not have $\pm C_{3} / R^{3}-C_{6} / R^{6}$ behaviour but rather more complex shapes. In addition to this, $C_{3}$ coefficients are much smaller at $6 p+5 s$ asymptotes since they are proportional to oscillator strength, whereas $C_{6}$ coefficients are larger since they are proportional to $n^{4}$ (fourth power of the effective main quantum number).

Although the theoretical evaluation of broadening parameters would be useful for the comparison with the present experiment, that task is beyond the scope of the present paper.

Since the mean room temperature thermal velocity of atoms in the cell is such that the average time of flight between the cell windows is much smaller than the residence time of $\mathrm{Rb}$ atoms on a wall, there are much more atoms adsorbed on the walls than freely-moving atoms in the gas [18]. However, we believe that at higher temperatures (densities) the main contribution to our observed broadening and shift results from the dipole-dipole interaction of atomic vapour atoms, together with some additional effects already mentioned in this section.

\section{Conclusion}

We demonstrated that a nano-cell with a thickness of $L=$ $\lambda / 2(210 \mathrm{~nm})$ is a convenient tool for the broadening rate and frequency shift measurements of the Rb $420 \mathrm{~nm}$ spectral line at relatively high atomic vapour densities up to $\sim 10^{17} \mathrm{~cm}^{-3}$. We discussed the problems connected with the origin of broadening and shift parameters. In addition, we pointed out the complexity of the hyperfine potential curves of the $\mathrm{Rb}(5 \mathrm{~s}, 6 \mathrm{p}, \mathrm{F})+\mathrm{Rb}(5 \mathrm{~s}, \mathrm{~F})$ homonuclear and heteronuclear interactions with both ${ }^{87} \mathrm{Rb}$ and ${ }^{85} \mathrm{Rb}$ isotopes involved in the construction of the broadening and shift in the bulk vapour of the thin cell.

\section{Acknowledgments}

We thank A Sarkisyan for his valuable participation in the fabrication of the nano-cell as well as D Bloch for lucrative discussions. Also, we wish to acknowledge the help from Ticijana Ban who carefully read the final version of the paper. This work was supported, in part, by ANSEF grant PSnano-657 and SCOPES grant IB7320-110684/1 and INTAS South-Caucasus grant 06-1000017-9001. The work was also supported by Ministry of Science, Education and Sports of the Republic of Croatia under the project number 035-03528512857.

\section{References}

[1] Briaudeau S, Bloch D and Ducloy M 1999 Phys. Rev. A 593723 and references therein

[2] Sarkisyan D, Bloch D, Papoyan A and Ducloy M 2001 Opt. Commun. 200201

[3] Dutier G, Yarovitski A, Saltiel S, Papoyan A, Sarkisyan D, Bloch D and Ducloy M 2003 Europhys. Lett. 6335

[4] Sarkisyan D, Varzhapetyan T, Sarkisyan A, Malakyan Yu, Papoyan A, Lezama A, Bloch D and Ducloy M 2004 Phys. Rev. A 69065802

[5] Aumiler D, Ban T and Pichler G 2004 Phys. Rev. A 70032723

[6] Failache H, Saltiel S, Fichet M, Bloch D and Ducloy M 1999 Phys. Rev. Lett. 835467

[7] Nesmeyanov A N 1963 Vapor Pressure of Elements (New York: Academic)

[8] Atiola A, Gibson-Wilde B C, Lindsay A C, Nicol J L and Whittingham I B 1988 J. Phys. B: At. Mol. Opt. Phys. 21249

[9] Walkup R, Stewart B and Pritchard D E 1984 Phys. Rev. A 29169

[10] Ping W, Gallagher A and Cooper J 1997 Phys. Rev. A 561598

[11] Gorris-Neveux M, Monnot P, Fichet M, Ducloy M, Barbé R and Keller J C 1997 Opt. Commun. 13485

[12] Fichet M et al 2007 Europhys. Lett. 7754001 and references therein

[13] Bloch D and Ducloy M 2005 Atom-Wall Interaction Advances in Atomic, Molecular and Optical Physics vol 50 ed B Bederson and H Walther (San Diego, CA: Elsevier) pp 91-156

[14] Jabbour Z J, Sagle R, Namiotka R K and Huennekens J 1995 J. Quant. Spectrosc. Radiat. Transfer 54767

[15] Guo J, Cooper J and Gallagher A 1996 Phys. Rev. A 531130

[16] Kemmann M, Mistrik I, Nussmann S, Helm H, Williams C and Julienne P S 2004 Phys. Rev. A 69022715

[17] Weiner J, Bagnato V S, Zilio S and Julienne P S 1999 Rev. Mod. Phys. 711

[18] Ducloy M 2004 Europhys. News 3540 